\title{
An Optimal Virtual Inertia Controller to Support Frequency Regulation in Autonomous Diesel Power Systems with High Penetration of Renewables
}

\author{
Miguel Torres and Luiz A.C. Lopes \\ Power Electronics and Energy Research Group \\ Department of Electrical and Computer Engineering \\ Concordia University, Montreal, Quebec, Canada H4G 2M1 \\ Phone: +1-514-8482424 (ext. 3080), e-mail: \{mi_torre, lalopes\}@ece.concordia.ca
}

\begin{abstract}
This paper addresses the problem of frequency control in autonomous diesel-based power system with high penetration of renewables. Usually, small power systems with high penetration of renewable energies are supplied by one or two small diesel generators, resulting in a system with a relatively low moment of inertia, and which can be susceptible to significant frequency variations. However, frequency regulation can be supported by modifying the inertial response of the system in an artificial way, i.e., by adding a virtual inertia. The latter can be performed by controlling the power electronics interface of a distributed generator or an energy storage unit. In this work, a controller is designed to provide the optimal virtual inertia which minimizes, according to the proposed performance index, variations in the fundamental frequency as well as in the power flow through the energy storage system. The optimal controller is compared by simulations with other virtual inertia control strategies.
\end{abstract}

\section{Keywords}

frequency regulation, virtual inertia, optimal control, energy storage, hybrid power system.

\section{Introduction}

Diesel generators (gensets) are very popular as main power source in stand-alone power systems. For instance, in autonomous wind-diesel power systems (AWDPS) [1], the power grid is established by a diesel generator-which is a controllable source of energy-and a wind generator (WG) is used to complement power production when the wind turbine reaches a specific speed. With an appropriate control strategy fuel consumption can be reduced, lowering the cost of the energy produced. However, aside from reducing fuel consumption, there also exists the necessity of controlling the frequency of the voltage being supplied to the load. The latter is specially important in small power systems $(10-200 \mathrm{~kW})$ with high penetration of renewable energies [2], [3]. Usually, this kind of grids are supplied by one or two small gensets, resulting in a system with a relatively low moment of inertia, and which can be susceptible to significant frequency variations due to sudden variations in load demand and/or in the output power of a renewable energy source.

Virtual synchronous machine/generator (VSM, VISMA, VSG) is a novel concept and it has been proposed as a control strategy to tackle stability issues in power systems with a large fraction of inertia-less distributed generators [4]-[9]. The main idea is to control the power electronics interface (PEI) of a distributed generator, or energy storage unit, in order to behave as a conventional rotating generator, which consists of a prime mover and a synchronous machine [10]. Virtual inertia control is a particular case of a VSM implementation, where only the action of the prime mover is emulated to support frequency control.

\section{A. Interest of the work}

Since the fundamental concept of VSMs was formally introduced, it has been subject of several publications. Most of the research deals with specific issues on the implementation of VSMs such as: the necessary requirements of the PEI to perform a VSM [11], selection of the storage media [12], and laboratory prototypes for experimental tests [13]. The most recent literature related to VSMs found on the IEEE database has been reviewed and, to the best of our knowledge, no research work has been reported concerning optimal control.

\section{B. Objectives}

1) Main objective. Design an optimal controller for the ESS in order to emulate a virtual inertia to support frequency control in a diesel based power system.

2) Secondary objectives. i) Model main components of the system, ii) Compare the performance of the optimal controller versus other virtual inertia controllers, and iii) analyze the effects on the ESS of using the optimal controller.

\section{Main contribution}

The optimization of the virtual inertia controller with the performance index being the integrated sum of the weighted quadratic regulating errors (QRE) of the power frequency and the power flow through the ESS.

\section{System overview and modeling}

The system of this study is an autonomous power system based on a diesel generator and a wind generator connected in parallel (see Fig. 1). It also has an energy storage system which is interfaced to the ac-bus by a bidirectional power electronics interface. The active 


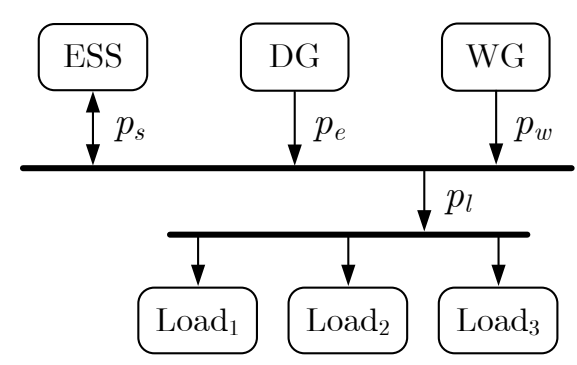

Fig. 1. Power system.

power balance of the system is governed by the following equation:

$$
p_{e}+p_{w}+p_{s}=p_{l} \text {. }
$$

\section{A. Load}

The load represents the uncontrolled end-user power demand and it is considered as a disturbance signal $p_{l}$.

\section{B. Wind generator}

The WG used in this work is a fixed-speed fixed-pitch (FSFP) configuration. Main advantages of this configuration are its simplicity and the robustness of the squirrel cage induction generator (SCIG). On the other hand, the main disadvantages comes from the fact that the SCIG operates over a narrow range around the synchronous speed. More details about this configuration and comparisons with other conversion schemes can be found on [14]. The modeling of the WG is based on equations presented in [3]. A conceptual block diagram of the model is shown in Fig. 2 and each of its components are herewith explained.

1) Wind speed. Wind speed, $v_{w}$, is defined by four components:

$$
v_{w}=V_{w b}+v_{w r}+v_{w g}+v_{w t}
$$

where, $V_{w b}$ is the base wind component, $v_{w r}$ the ramp component, $v_{w g}$ the gust component, and $v_{w t}$ the turbulence component. The ramp component is defined as:

$$
v_{w r}(t)= \begin{cases}\frac{V_{w r}}{t_{2 r}-t_{1 r}} t & , t_{1 r} \leq t \leq t_{2 r}, \\ 0 & \text { otherwise. }\end{cases}
$$

the gust component as:

$$
v_{w g}(t)= \begin{cases}\frac{V_{w g}}{2}(1-\cos \varphi) & , t_{1 g} \leq t \leq t_{2 g} \\ 0 & \text { otherwise. }\end{cases}
$$

where $\varphi=2 \pi\left(t-t_{1 g}\right) /\left(t_{2 g}-t_{1 g}\right)$. Finally, the turbulence component is defined in terms of its power spectral density (PSD):

$$
v_{w t}=2 \sum_{k=1}^{N}\left\{S\left(\omega_{k}\right) \delta \omega\right\}^{\frac{1}{2}} \cos \left(\omega_{k} t+\phi_{k}\right)
$$

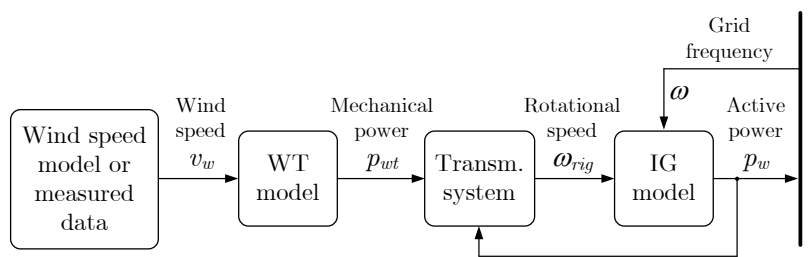

Fig. 2. Wind generator conceptual block diagram.

where $\omega_{k}=(k-1 / 2) \delta \omega$ and $\phi_{k}=\operatorname{rand}(0,2 \pi)$ are the frequency and phase of the $k$-th cosinusoidal component, and $S\left(\omega_{k}\right)$ is the spectral density function defined as:

$$
S\left(\omega_{k}\right)=\frac{2 z_{0} F^{2}\left|\omega_{k}\right|}{\pi^{2}\left\{1+\left(\frac{F \omega_{k}}{\bar{v} \pi}\right)^{2}\right\}^{\frac{4}{3}}}
$$

2) Wind turbine. The model of the wind turbine consists of two elements: a first order system (7) which represents the filtering of the high frequency components in the wind speed by the turbine, and the output power equation of the wind turbine (8).

$$
\begin{aligned}
\tau_{w} \dot{v}_{w f} & =-v_{w f}+v_{w} \\
p_{w t} & =\frac{1}{2} \rho \pi R_{w t}{ }^{2} c_{p}(\lambda) v_{w f}{ }^{3}
\end{aligned}
$$

where, $\tau_{w}$ is the time constant which depends on the turbine size, $v_{w f}$ is the filtered wind speed, $\rho$ is the air density, $R_{w t}$ is the radius of the turbine rotor, and $c_{p}(\lambda)$ is the performance factor of the turbine given by:

$$
c_{p}(\lambda)=c_{1}\left\{c_{2}\left(\frac{1}{\lambda}-c_{5}\right)-c_{3}\right\} e^{-c_{4}\left(\frac{1}{\lambda}-c_{5}\right)}
$$

with $\lambda$ being the tip speed ratio:

$$
\lambda=\frac{R_{w t} \omega_{w t}}{v_{w f}}
$$

where $\omega_{w t}$ is the rotational speed of the WT. Coefficients $c_{1 . .5}$ are defined later to fit the characteristic curve of the turbine of interest.

3) Transmission system. The transmission system consists of the high speed shaft (IG side) and the low speed shaft (WT side) connected together by a gear box. The model considered is the following:

$$
J_{w} \dot{\omega}_{r i g}=\frac{1}{N_{g b}} T_{w t}-T_{i g}
$$

where, $J_{w}$ is the lumped inertia of rotating parts, $N_{g b}$ is the conversion ratio of the gear box, $T_{w t}$ the mechanical torque of the turbine, $T_{i g}$ the electromagnetic torque of the induction generator, and $\omega_{\text {rig }}$ is the rotor speed of the induction generator. Now, the rotational speed of the WT can be defined as

$$
\omega_{w t}=\frac{\omega_{r i g}}{N_{g b}}
$$

4) Induction generator. The proposed model for the IG is based on the fact that the generator operates in a small 
region around the synchronous speed $\omega_{\text {sync }}[15]$, then the torque can be approximated by:

$$
T_{i g} \approx \frac{3 V_{t}^{2}\left(\omega_{\text {rig }}-\omega_{\text {sync }}\right)}{\omega_{\text {sync }}^{2} R_{2}^{\prime}}
$$

where, $V_{t}$ is the terminal voltage, $R_{2}^{\prime}$ is the rotor resistance, and $\omega_{\text {sync }}$ is the synchronous speed.

\section{Diesel generator}

The diesel generator model consists of two main components coupled by a common shaft: the synchronous generator (SG) and the diesel prime mover. Due to the scope of this work, the electrical dynamics of the SG are neglected. Also, in order to focus the study on frequency variations, it is assumed that the amplitude of grid voltages are kept constant by the automatic voltage regulator (AVR) of the SG. Therefore, the proposed model for the diesel generator and its governor can be represented by the following equations:

$$
\begin{aligned}
\Delta \dot{\omega}_{r} & =-\frac{\left(k_{f}+k_{d}\right)}{J} \Delta \omega_{r}+\frac{1}{J} \Delta T_{m}-\frac{1}{J} \Delta T_{e} \\
\Delta \dot{T}_{m} & =-\frac{1}{\tau_{e}} \Delta T_{m}+\frac{k_{e}}{\tau_{e}} \Delta u_{e}\left(t-t_{d}\right) \\
\Delta \dot{u}_{e} & =-k_{c} \Delta \omega_{r}
\end{aligned}
$$

where $\omega_{r}$ is the SG rotor speed, $u_{e}$ is the control signal from the speed regulator (governor) to the fuel injection system, $T_{m}$ is the mechanical torque provided by the diesel engine at the common shaft, and $T_{e}$ is the electromechanical torque produced at the common shaft due to the active power balance of the grid (1). The system has the following parameters: $J$ the lumped inertia of rotating parts, $k_{f}$ the friction coefficient of the shaft, $k_{d}$ the SG damping torque coefficient, $\tau_{e}$ and $k_{e}$ the fuel injection system time constant and gain, $t_{d}$ the engine dead time which represents the elapsed time until torque is produced at the engine shaft, and $k_{c}$ the speed regulator gain (see block diagram in Fig. 3 .

\section{Energy storage system}

The ESS consists of two main components: the PEI and the storage media. The PEI is a two-level VSC and the storage media is considered as an ideal dc voltage source, $V_{d c}$, connected to the dc-bus of the VSC. If the abc-dq transformation is perfectly synchronized with grid voltages, the output active power flow can be defined as:

$$
\Delta p_{s}=V_{d} \Delta i_{s}^{d}
$$

where $V_{d}$ and $i_{s}^{d}$ are the d-axis component of the the grid voltages and VSC ac currents, respectively. From (17) it can be seen that the active power of the VSC can be controlled by means of $i_{s}^{d}$, whose dynamic is given by:

$$
L \Delta \dot{\hat{i_{s}^{d}}}=-r \Delta i_{s}^{d}+2 \pi f L \Delta i_{s}^{q}+V_{d c} \Delta m^{d}-V_{d}
$$

where $i_{s}^{q}$ is the q-axis component of the VSC ac currents, $m^{d}$ is the d-component of the modulation indexes, $f$ is the grid frequency, $L$ and $r$ are the inductance and resistance of the output filter of the VSC. To decouple and operate $i_{s}^{d}$ in closed loop, the following control law is defined:

$$
\begin{aligned}
\Delta m^{d}=\frac{1}{V_{d c}}\{ & \left(k_{s i}+r\right) \Delta i_{s r e f}^{d} \\
& \left.\quad-k_{s i} \Delta i_{s}^{d}-2 \pi f L \Delta i_{s}^{q}+V_{d}\right\}
\end{aligned}
$$

where, $k_{s i}$ is the current controller gain, and $i_{s \text { ref }}^{d}$ is the d-axis current reference signal (see block diagram in Fig. 3). For more details on the modeling and control of a VSC, refer to [16].

\section{Optimal virtual inertia control}

\section{A. Virtual inertia concept}

If the active power through the PEI of the ESS is controlled in inverse proportion to the derivative of the grid frequency, we are emulating a virtual inertia in the power system, thus enhancing the inertial response to changes in the power demand. Then, the control law for the active power of the VSC will be:

$$
\Delta p_{\text {s ref }}=-k_{v i}{k_{r}}^{2} f_{o} \Delta \dot{f}
$$

where $k_{r}=4 \pi / n_{p}$ is the conversion factor between rotor speed and grid frequency with $n_{p}$ being the number of poles of the machine, $k_{v i}$ is the virtual inertia added to the system, and $f_{o}$ is the nominal grid frequency (see block diagram in Fig. 3 .

\section{B. Optimal controller}

Instead of being constant, the virtual inertia $k_{v i}$ can be designed to be optimal for a given design specification. In order to find such optimal value, we will use the deterministic linear quadratic regulator (LQR) approach. For details on the theoretical framework and mathematical derivations related to the LQR problem refer to [17]-[19]. For the design of the optimal controller, consider the system in the form:

$$
\Delta \dot{f}=a \Delta f+b \Delta p_{s}
$$

with $a=-\left(k_{d}+k_{f}\right) / J$ and $b=1 /\left(J \omega_{r o} k_{r}\right)$. The general formulation of the LQR problem requires to define a mathematical performance criterion, often called performance index or function cost. Then, the proposed performance index is defined as the integrated sum of the weighted quadratic regulating errors of the grid frequency and the ESS power (22). By doing this, we are looking for minimizing the frequency variations, but since this is accomplished by the PEI of the ESS, at the same time we penalize - by means of the weighting factor $\alpha$ the amount of power flowing through the ESS. The expression for the performance index is:

$$
I=\int_{0}^{\infty}\left\{\Delta f^{2}(t)+\alpha \Delta p_{s}{ }^{2}(t)\right\} d t
$$

where $\alpha$ is the weighting factor. Therefore, the control input which minimizes $I$ is defined as:

$$
\Delta p_{s}{ }^{\star}=-\frac{1}{\alpha} b F \Delta f
$$




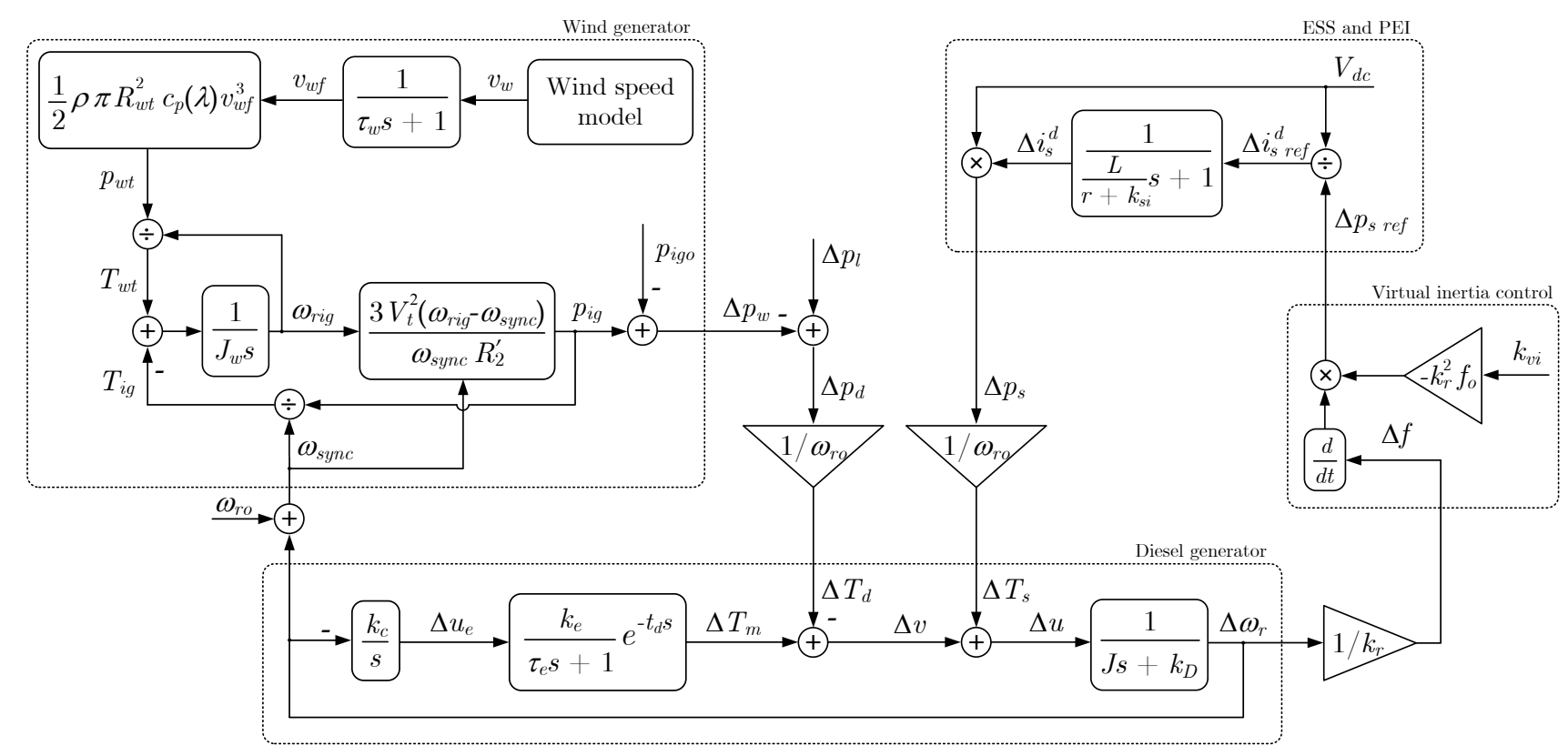

Fig. 3. System model block diagram and control scheme.

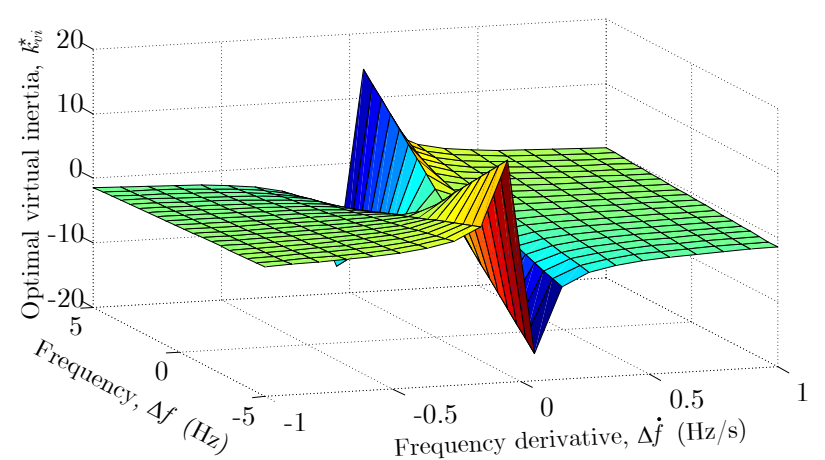

Fig. 4. Optimal virtual inertia surface for $\gamma=1$.

with $F$ being the positive solution of the stationary form of the Riccati equation:

$$
F^{2}-\frac{2 a \alpha}{b^{2}} F-\frac{\alpha}{b^{2}}=0
$$

Finally, from (23) the optimal virtual inertia can be defined as:

$$
k_{v i}^{\star}=\gamma \frac{\Delta f}{\Delta \dot{f}}
$$

where the factor

$$
\gamma=\frac{1}{k_{r}^{2} f_{o}}\left\{\frac{a}{b}+\sqrt{\left(\frac{a}{b}\right)^{2}+\frac{1}{\alpha}}\right\}
$$

has been defined to simplify the notation. The surface of Fig. 4 represents the optimal values of $k_{v i}$, with $\gamma=1$, for each pair $(\Delta \dot{f}, \Delta f) \in[-1,1] \times[-5,5]$. Fig. 4 can also be used to understand how the optimal controller operates, e.g., Fig. 5 shows the plot of $k_{v i}^{\star}(\gamma=0.5,1,2)$ when $\Delta f>0$ and $\Delta f<0$. For the case when $\Delta f>0$ : if $\Delta \dot{f}$ tries to increase, the optimal controller increases the virtual inertia to slow down the frequency excursion; and the opposite occurs when $\Delta \dot{f}$ tries to decrease. The same analysis can be done for the case when $\Delta f<0$. Fig. 6 shows the dynamic response of the controller for

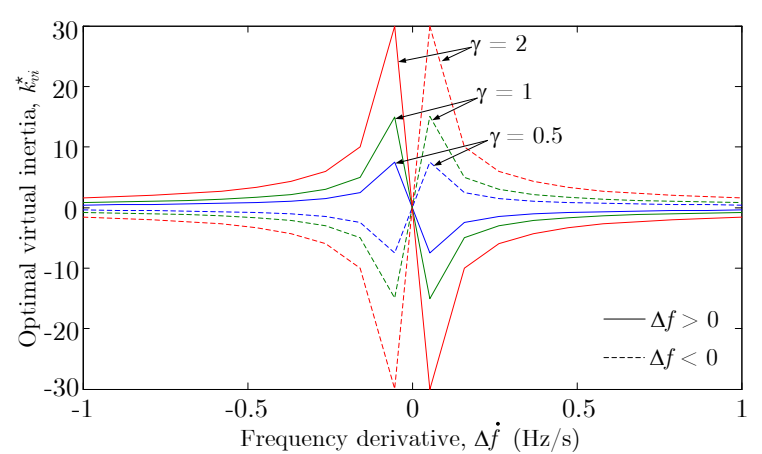

Fig. 5. Optimal virtual inertia.

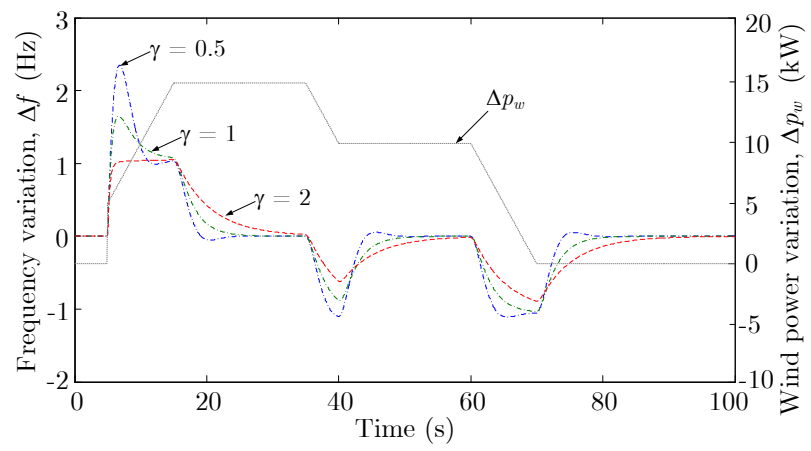

Fig. 6. Frequency response for different values of $\gamma$.

different values of parameter $\gamma$. From (26) it can be noted that the controller gain $\gamma$ and weighting factor $\alpha$ are inversely related, i.e., a higher value of the controller gain $\gamma$ will decrease the value of the weighting factor $\alpha$ in (22). The latter implies that, as $\gamma$ increases, larger variations in $\Delta p_{s}$ will be tolerated (see Fig. 7).

\section{Performance verification}

The optimal controller is compared with the following cases: $i)$ no virtual inertia, $k_{v i}=0$; ii) constant virtual 


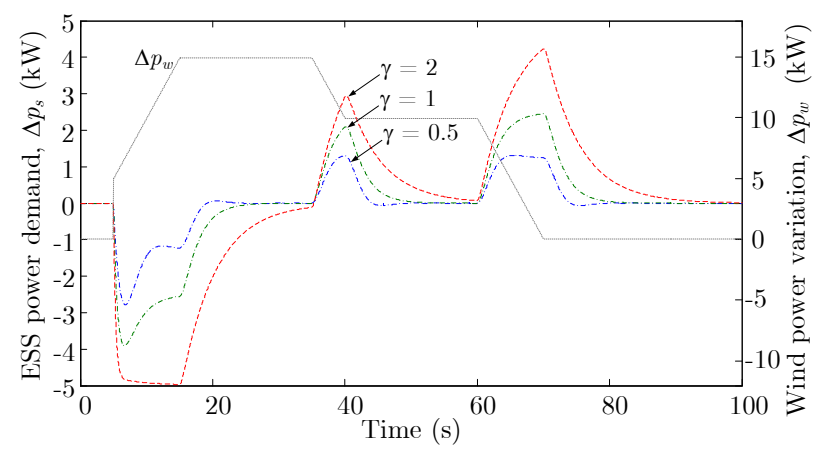

Fig. 7. ESS power demand for different values of $\gamma$.

inertia, $k_{v i}=\bar{k}_{v i}$; and iii) a modified virtual inertia defined as:

$$
\widetilde{k}_{v i}= \begin{cases}\bar{k}_{v i} & \text { if } \Delta f \Delta \dot{f} \geq 0 \\ 0 & \text { otherwise }\end{cases}
$$

The system is simulated for a 100s wind speed profile and a constant load demand. The wind profile consists of a wind gust at $t=20 \mathrm{~s}$ followed by a positive ramp. All the parameters used in simulations are summarized in Tab. I] Tab. II] Tab. [II], Tab. IV] Tab. V. Tab. VI, and presented in the appendix section. The simulated wind speed is shown on Fig. 8 Fig. 9 shows the output power of the wind turbine, which is the result of its interaction with the wind and with the grid frequency, in this case. Fig. 10 shows the frequency variation in the system, for different conditions of virtual inertia. Finally, Fig. 11 shows the variations in the power flow of the ESS, which is the one that emulates the virtual inertia.

As can be seen from Fig. 10, when the system is operated with constant inertia $\left(k_{v i}=0\right.$ and $\left.k_{v i}=\bar{k}_{v i}\right)$ the inertial response is better at the beginning of the perturbation (less reactive to changes) but the oscillations last for longer time, since there is no additional damping in the system. At the same time, as the constant virtual inertia increases, the peak power through the ESS increases as well. The latter has direct influence on the necessary power ratings of the storage media and its power converter.

In the case when the system is operated with variable virtual inertia $\left(k_{v i}=\widetilde{k}_{v i}\right.$ and $\left.k_{v i}=k_{v i}^{\star}\right)$ it is possible to say that the response is better than in the case of constant inertia. However, since control law (27) is piecewise constant, each change of condition represents a fast commutation from one power level to another, which becomes into an operational requirement for the storage device.

Compared with the proposed cases, the optimal controller presents a good performance in terms of, oscillation mitigation, peak frequency, and power consumption. For instance, consider Fig. 10 and the case of the optimal controller vs. control law (27): both operate within the power range $[-7,5] \mathrm{kW}$, however the optimal controller achieves almost $50 \%$ less in peak frequency deviation and it settles back at least 3 times faster. It also has the advantage of having just one parameter for tuning, $\gamma$.

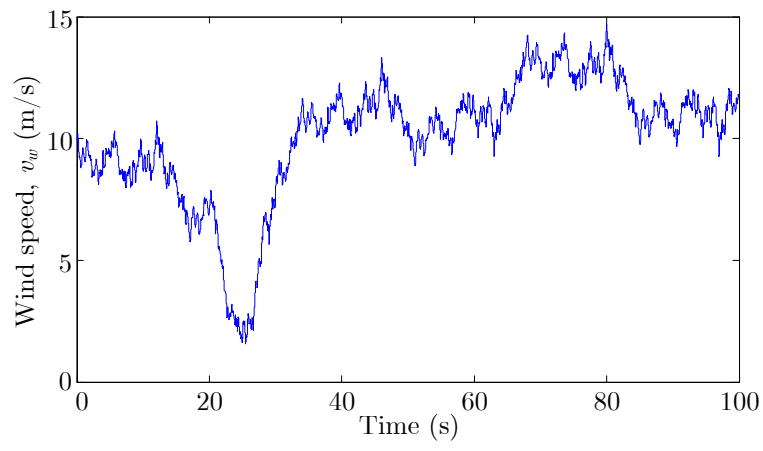

Fig. 8. Wind speed.

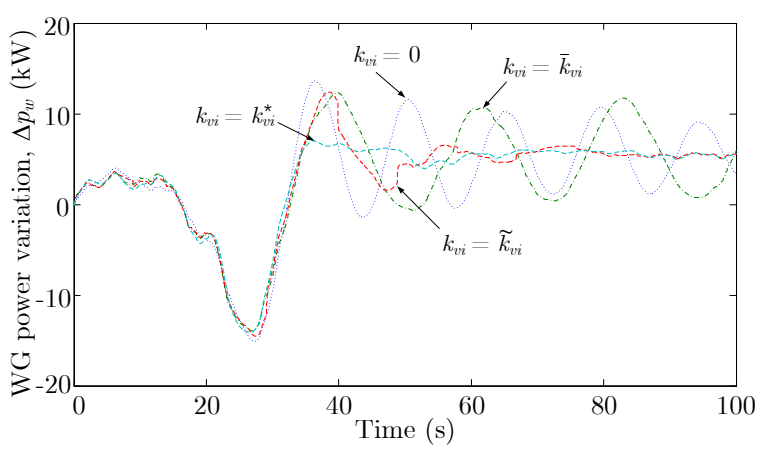

Fig. 9. Comparison of WG output power variations.

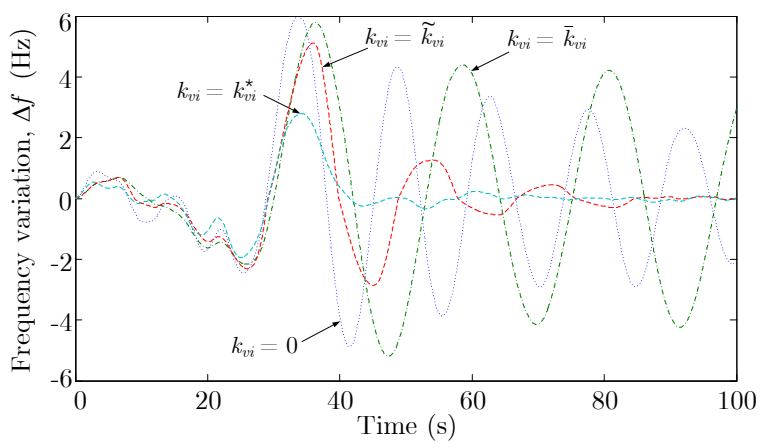

Fig. 10. Comparison of frequency response for different virtual inertia controllers.

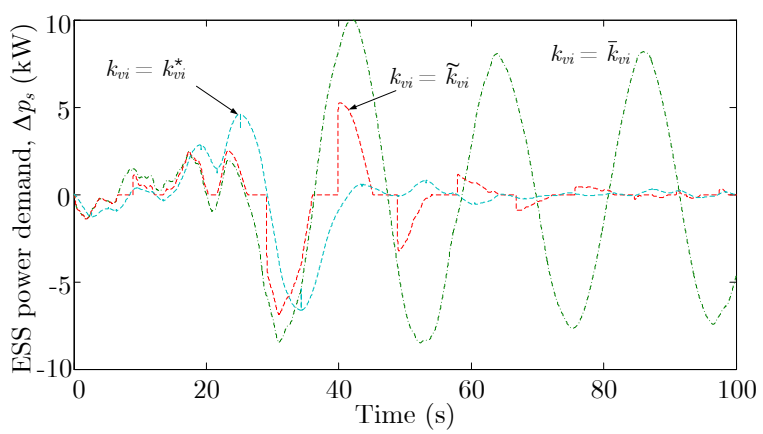

Fig. 11. Comparison of power usage for different virtual inertia controllers.

\section{Conclusion}

In this work, we presented the design of an optimal controller for the emulation of virtual inertia to support frequency regulation in a diesel based power system. The model of each main component of the system was developed. The performance of the optimal controller was compared with other controllers by simulation. The 
results show a good performance of the optimal controller in minimizing frequency variations, in exchange of power consumption/injection from/to the ESS. By adjusting only one parameter of the controller, it is possible to change its control effort, which has direct influence on the ESS power flow requirements. The possibility to include constraints in the formulation of the optimization problem, to satisfy physical limitations of the system, should be considered as future work.

\section{Appendix: simulation parameters}

Table I. - Diesel generator model

\begin{tabular}{|l|c|c|c|}
\hline Parameter description & Symbol & Value & Unit \\
\hline Nominal power & $P_{e n}$ & 30 & $\mathrm{~kW}$ \\
\hline Number of poles & $n_{p}$ & 2 & \\
\hline Moment of inertia & $J$ & 0.9 & $\mathrm{~kg} \mathrm{~m}^{2}$ \\
\hline Damping coefficient & $k_{D}$ & 0.4 & $\mathrm{~kg} \mathrm{~m}^{2} \mathrm{~s}^{-1}$ \\
\hline Fuel injection gain & $k_{e}$ & 1 & \\
\hline Fuel injection time constant & $\tau_{e}$ & 0.2 & $\mathrm{~s}$ \\
\hline Diesel engine delay & $\tau_{d}$ & 0.011 & $\mathrm{~s}$ \\
\hline Speed controller gain & $k_{c}$ & 0.4 & \\
\hline
\end{tabular}

Table II. - Wind generator model

\begin{tabular}{|l|c|c|c|}
\hline Parameter description & Symbol & Value & Unit \\
\hline Nominal power & $P_{w n}$ & 20 & $\mathrm{~kW}$ \\
\hline Number of poles & $n_{p}$ & 2 & \\
\hline Moment of inertia & $J_{w}$ & 1.2 & $\mathrm{~kg} \mathrm{~m}^{2}$ \\
\hline Terminal voltage & $V_{t}$ & 400 & $\mathrm{~V}$ \\
\hline Rotor resistance & $R_{2}^{\prime}$ & 0.221 & $\Omega$ \\
\hline Air density & $\rho$ & 1.275 & $\mathrm{~kg} \mathrm{~m}^{-3}$ \\
\hline Rotor radius & $R_{w t}$ & 5 & $\mathrm{~m}$ \\
\hline Gear box ratio & $N_{g b}$ & 40 & \\
\hline Wind speed filter time constant & $\tau_{w}$ & 1 & $\mathrm{~s}$ \\
\hline
\end{tabular}

Table III. - Performance factor coefficients

\begin{tabular}{|l|c|c|c|c|c|}
\hline Wind generator type & $\mathbf{c}_{\mathbf{1}}$ & $\mathbf{c}_{\mathbf{2}}$ & $\mathbf{c}_{\mathbf{3}}$ & $\mathbf{c}_{\mathbf{4}}$ & $\mathbf{c}_{\mathbf{5}}$ \\
\hline Fixed-speed fixed-pitch & 0.76 & 125 & 6.94 & 16.5 & -0.002 \\
\hline
\end{tabular}

Table IV. - Wind speed model

\begin{tabular}{|l|c|c|c|}
\hline Parameter description & Symbol & Value & Unit \\
\hline Base wind speed & $V_{w b}$ & 8 & $\mathrm{~m} / \mathrm{s}$ \\
\hline Ramp amplitude & $V_{w r}$ & 4 & $\mathrm{~m} / \mathrm{s}$ \\
\hline Ramp start time & $t_{1 r}$ & 20 & $\mathrm{~s}$ \\
\hline Ramp end time & $t_{2 r}$ & 60 & $\mathrm{~s}$ \\
\hline Gust amplitude & $V_{w g}$ & -3 & $\mathrm{~m} / \mathrm{s}$ \\
\hline Gust start time & $t_{1 g}$ & 20 & $\mathrm{~s}$ \\
\hline Gust end time & $t_{2 g}$ & 30 & $\mathrm{~s}$ \\
\hline PSD components & $N$ & 100 & \\
\hline PSD frequency step & $\delta \omega$ & 0.37 & $\mathrm{rad} / \mathrm{s}$ \\
\hline Landscape roughness coefficient & $z_{0}$ & 0.004 & \\
\hline Turbulence length scale & $F$ & 400 & $\mathrm{~m}$ \\
\hline Mean wind speed at reference height & $\bar{v}$ & 8 & $\mathrm{~m} / \mathrm{s}$ \\
\hline
\end{tabular}

Table V. - Virtual inertia controller

\begin{tabular}{|l|c|c|}
\hline Parameter description & Symbol & Value \\
\hline Constant virtual inertia & $\bar{k}_{v i}$ & $3 J$ \\
\hline Optimal virtual inertia gain & $\gamma$ & 1 \\
\hline
\end{tabular}

Table VI. - Power electronics interface

\begin{tabular}{|l|c|c|c|}
\hline Parameter description & Symbol & Value & Unit \\
\hline Filter inductance & $L$ & 32 & $\mathrm{mH}$ \\
\hline Filter resistance & $r$ & 0.2 & $\Omega$ \\
\hline Current controller gain & $k_{s i}$ & 10 & \\
\hline
\end{tabular}

\section{Acknowledgment}

The authors would like to thank the financial support of the Chilean Government, through the National Commission for Science and Technology Research (CONICYT), and the Faculty of Electrical Engineering and Computer Science of Concordia University.

\section{References}

[1] H. Nacfaire, Ed., Wind-diesel and wind autonomous energy systems. Elsevier Applied Science, 1989.

[2] J. Morren, S. de Haan, and J. Ferreira, "Contribution of DG units to primary frequency control," in Future Power Systems, 2005 International Conference on, Nov. 2005, pp. 1-6.

[3] T. Ackermann, Ed., Wind Power in Power Systems. John Wiley \& Sons, Ltd., Mar. 2005.

[4] H. P. Beck and R. Hesse, "Virtual synchronous machine," in Electrical Power Quality and Utilisation, 2007. EPQU 2007. 9th International Conference on, Oct. 2007, pp. 1-6.

[5] K. Visscher and S. De Haan, "Virtual synchronous machines for frequency stabilisation in future grids with a significant share of decentralized generation," in SmartGrids for Distribution, 2008 IET-CIRED. CIRED Seminar, Jun. 2008, pp. 1-4.

[6] J. Driesen and K. Visscher, "Virtual synchronous generators," in Power and Energy Society General Meeting - Conversion and Delivery of Electrical Energy in the 21st Century, 2008 IEEE, Jul. 2008, pp. 1-3.

[7] S. de Haan, R. van Wesenbeeck, and K. Visscher. (2008, Oct.) VSG control algorithms: present ideas. http://www.vsync.eu/fileadmin/vsync/user/docs/20080728VSYNC-General_presentation.pdf. Project VSYNC. [Online] Available: http://www.vsync.eu

[8] Q.-C. Zhong and G. Weiss, "Static synchronous generators for distributed generation and renewable energy," in Power Systems Conference and Exposition, 2009. PSCE'09. IEEE/PES, Mar. 2009 , pp. 1-6.

[9] M. Van Wesenbeeck, S. de Haan, P. Varela, and K. Visscher, "Grid tied converter with virtual kinetic storage," in PowerTech, 2009 IEEE Bucharest, Jul. 2009, pp. 1-7.

[10] I. Boldea, Synchronous Generators Handbook. CRC Press, 2006.

[11] T. Loix, S. De Breucker, P. Vanassche, J. Van den Keybus, J. Driesen, and K. Visscher, "Layout and performance of the power electronic converter platform for the VSYNC project," in PowerTech, 2009 IEEE Bucharest, Jul. 2009, pp. 1-8.

[12] M. Albu, K. Visscher, D. Creanga, A. Nechifor, and N. Golovanov, "Storage selection for DG applications containing virtual synchronous generators," in PowerTech, 2009 IEEE Bucharest, Jul. 2009, pp. 1-6.

[13] V. Van Thong, A. Woyte, M. Albu, M. Van Hest, J. Bozelie, J. Diaz, T. Loix, D. Stanculescu, and K. Visscher, "Virtual synchronous generator: Laboratory scale results and field demonstration," in PowerTech, 2009 IEEE Bucharest, Jul. 2009, pp. 1-6.

[14] H. Li and Z. Chen, "Overview of different wind generator systems and their comparisons," Renewable Power Generation, IET, vol. 2, no. 2, pp. 123-138, Jun. 2008.

[15] M. A. El-Sharkawi, Fundamentals of electric drives. Toronto: Cengage Learning, 2000.

[16] J. Espinoza, "Inverters," in Power Electronics Handbook, M. H. Rashid, Ed. San Diego-California, USA: Academic Press, 2007, ch. 15 , pp. 353-402.

[17] R. C. K. Lee, Optimal estimation, identification, and control. Cambridge: M.I.T. Press, 1964, vol. 28.

[18] M. Athans and P. L. Falb, Optimal control;an introduction to the theory and its applications. New York: McGraw-Hill, 1966.

[19] H. Kwakernaak and R. Sivan, Linear optimal control systems. New York: Wiley Interscience, 1972. 